\title{
Current concepts on the management of tooth wear: part 3. Active restorative care 2: the management of generalised tooth wear
}
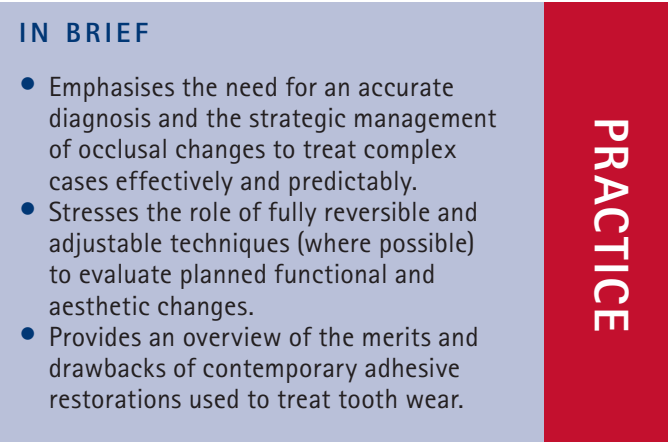

\author{
S. B. Mehta, ${ }^{1}$ S. Banerji, ${ }^{2}$ B. J. Millar ${ }^{3}$ and J.-M. Suarez-Feito ${ }^{4}$ \\ VERIFIABLE CPD PAPER
}

Paper 3 of this series on the current concepts of tooth wear management will focus on the provision of active restorative intervention for cases presenting with generalised tooth wear. The use of both contemporary adhesive and traditional conventional techniques applied to treat cases of generalised tooth wear will be discussed, including a consideration of the merits and drawbacks of each approach respectively.

\section{INTRODUCTION}

\section{Generalised tooth wear}

The principles for the management of cases presenting with generalised tooth wear (full arch or both arches respectively) follow the same basic tenets as outlined for localised wear cases in papers 1 and 2 of this series respectively. ${ }^{1,2}$ Hence, following

\section{CURRENT CONCEPTS ON THE MANAGEMENT OF TOOTH WEAR}

1. Assessment, treatment planning and strategies for the prevention and the passive management of tooth wear

2. Active restorative care 1 : the management of localised tooth wear

3. Active restorative care 2 : the management of generalised tooth wear

4. An overview of the restorative techniques and dental materials commonly applied for the management of tooth wear

\footnotetext{
1,2General Dental Practitioner and Senior Clinical Teacher, Department of Primary Dental Care, King's College London Dental Institute, Bessemer Road, London, SE5 9RW; ${ }^{3 *}$ Professor, Consultant in Restorative Dentistry, Department of Primary Dental Care, King's College London Dental Institute, Bessemer Road, London, SE5 9RW; ${ }^{4}$ Section of Periodontology, Faculty of Dentistry and Medicine, University of Oviedo, Oviedo, Spain

${ }^{*}$ Correspondence to: Professor Brian J. Millar Email: brian.millar@kcl.ac.uk
}

\section{Refereed Paper}

Accepted 14 November 2011

DOI: 10.1038/sj.bdj.2012.97

${ }^{\circledR}$ British Dental Journal 2012; 212: 121-127 the establishment of an accurate diagnosis and the identification of possible aetiological factors, an appropriate preventative regime should be implemented, usually followed by a period of passive monitoring. The purpose of the latter is primarily to ascertain the patient's compliance with the preventative programme, thus a reduction (or ideally) an elimination of the aetiological factors, to ensure that the process of active tooth wear has stopped (or considerably reduced) and finally to allow the dental operator to develop a rapport with the patient, and the patient to form a clear understanding of the complexities associated with the provision of active restorative care. While for some cases the elimination of possible causative factors may suffice, for a proportion of cases active restorative intervention will be required. The indications for active restorative intervention for a patient presenting with pathological tooth wear have been discussed in detail in paper $2 .^{2}$

For descriptive purposes, the restorative management of patients presenting with generalised tooth wear (TW) will be considered according to the three categories described by Turner and Missirilian, ${ }^{3}$ hence:

- Category 1 - excessive wear with loss of vertical dimension of occlusion

- Category 2 - excessive wear without loss of vertical dimension, but with space available
- Category 3 - excessive wear without loss of vertical dimension, but with limited space.

Regardless of the category, for any case of generalised TW where active intervention is being sought, a set of diagnostic casts mounted in centric relation (CR) is strongly advised.

While a semi-adjustable articulator with an arbitrary facebow may be considered to be acceptable, a kinematic transverse horizontal axis facebow transfer is preferable in helping to plan a tentative increase in the occlusal vertical dimension (OVD) without introducing errors in the horizontal jaw relationship. The true transverse horizontal axis may be significantly different anatomically from that determined with the use of an arbitrary facebow. ${ }^{4}$ In such cases, an alteration in the OVD will result in a loss of accuracy in the horizontal jaw relationship, culminating in erroneous restorations.

The desired increase in OVD will primarily be determined by what is necessary to produce functionally stable, aesthetic dental restorations and an adequate freeway space. Clinically this can be estimated by measurement of the existing OVD of the worn dentition and the face height with the mandible at rest with an adequate lip seal; the difference between the two measurements needs to accommodate the desired increase in the OVD and the freeway space. Once this 


\section{PRACTICE}
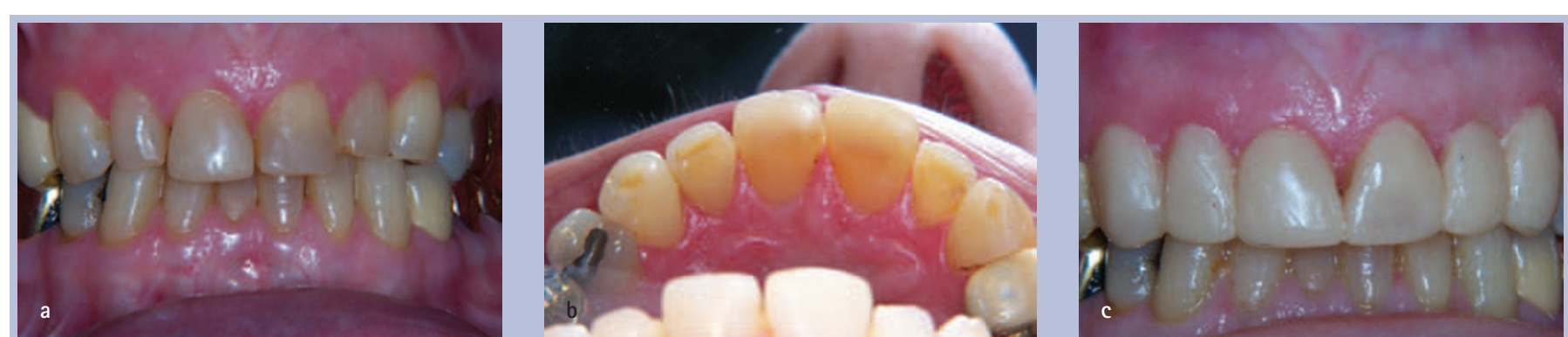

Figs 1a-c Composite resin 'mock-up' to demonstrate proposed aesthetic and functional changes to a worn dentition. Warmed resin composite (Gradia Direct, GC), has been applied to a worn dentition with the aid of a thermoplastic template, without any hard tissue conditioning

has been determined, the planned increase may be programmed into the articulator (by raising the pin on the articulator), and a diagnostic wax up fabricated accordingly (preferably on duplicate casts).

The transfer of the prescribed increase in OVD to the patient's dentition should be initially done by the use of fully reversible and adjustable materials (such as resin composite), so as to determine the patient's tolerance and aesthetic acceptance of the proposed changes (although this may not always be required or indeed be possible). Once the latter have been ascertained and the prescription accepted, then the interim material can be substituted accordingly. This will not only permit an appropriate period of time to evaluate tolerance of the proposed changes, but will also avoid the propagation of potential errors which may arise where definitive longer term restorations designed extra-orally (at a new occlusal vertical dimension on an articulator) are placed clinically (without applying interim, short to medium term restorations), as centric relation cannot always be recorded with absolute accuracy.

The diagnostic wax up should take account of basic aesthetic principles, as discussed in Paper 1 (such as tooth shape, length, inclination and relationship of the incisal edge to the lip line). ${ }^{1}$ It should also aim to provide occlusal stability, based on the principles of a mutually protective occlusal scheme. ${ }^{5}$

In essence, the final occlusal scheme should provide:

- Simultaneous stable bilateral tooth contacts

- Centric relation (CR) coincident with centric occlusion (CO)

- Disclusion of the posterior teeth, upon lateral and protrusive mandibular movements

- Anterior teeth disclusion, when posterior teeth contact in maximum intercuspation

- Shared/even anterior guidance

- Canine guided occlusion, with planned group function upon loss of canine guidance (or where the canine tooth may be unsuitable as a guiding unit), with the absence of working or nonworking side occlusal interferences.

The diagnostic wax up should be presented to the dental patient, to permit an evaluation of the anatomy, size, contours and shape of definitive restorations. It will also help to explain the proposed occlusal changes.

It is often useful to have a vacuum formed matrix (thermoplastic template) produced from a duplicate cast of the diagnostic wax-up, which may be applied to the unrestored dentition, with an intervening provisional crown and bridge material in the matrix, to provide the patient with a rough visual of the restorative end point, particularly where there may be planned significant changes to the anterior dentition. Alternatively, resin composite 'mockups' may be placed directly to evaluate proposed changes. Depicted by Figure 1 is an example of such a 'composite resin mock up' applied to a worn dentition to display proposed aesthetic and functional changes.

Unrealistic expectations may be apparent at this stage, which may require further patient education or revision of the treatment plan.

\section{RESTORATIVE TECHNIQUES - ADHESIVE VERSUS CONVENTIONAL}

Before discussing the approach taken for the management of a patient presenting with generalised tooth wear, regardless of the category of wear as described above, it is worthwhile overviewing the possible restorative protocols which are usually applied, hence the adhesive 'additive approach' versus the conventional approach.

Conventional restorative techniques (those which depend on mechanical tooth preparation features to provide retention and resistance form) have traditionally been the mainstay for the management of tooth surface loss. In recent times, with improvements in adhesive technology and the availability of superior resin composites, adhesive retained restorations have become ever increasingly popular.

The choice of a particular technique will depend on a variety of factors as listed below. However, it is important to state that any single case may well involve a combination of techniques as depicted by the case examples described above.

- Conventional restorations will require the copious removal of sound dental hard tissue (from tooth structure which will have already been compromised by the process of TW). Adhesive preparations in contrast are minimally invasive

- Conventional, full coverage restorations have been associated with high risks of loss of pulp tissue vitality. Saunders and Saunders reported in 1998 that 19\% of crowned teeth among a Scottish subpopulation had radiographic signs of peri-radicular disease. ${ }^{6}$ The latter may be heightened among teeth affected by tooth surface loss, as their pulpal tissues may have already been 'stressed' by the process of tooth wear. Furthermore, iatrogenic pulp exposure is more likely among teeth which have been affected by the process of wear, by virtue of the pulp chamber being closer to the occlusal surface of the affected tooth

- Conventional tooth preparations are 
'irreversible'. There are risks that some patients may not be able to tolerate planned changes to their occlusal scheme. Adhesive restorations offer a large element of flexibility, as minimal tooth reduction is required to accommodate the latter forms of dental restoration

- Conventional restorations require careful tooth preparation to provide adequate resistance and retention form. The process of preparation may be hampered by a lack of dental hard tissue in the case of a worn dentition

- The success of adhesive restorations is dependent to a large extent on the presence of a copious quantity of high quality tooth enamel. However, in the case of conventional restorations, the process of tooth preparation may lead to the loss of any residual enamel, which will reduce the intrinsic strength of the tooth and compromise the longevity of the restoration. According to a study by Edelhoff and Sorenssen, ${ }^{7}$ between $63 \%$ and $72 \%$ of coronal tooth tissue may removed during the preparation of a tooth to receive an all-ceramic or porcelain fused to metal crown

- Adhesive techniques are highly operative sensitive and require meticulous moisture control

- Conventional techniques are dependent on the need for provisional restorations. They are seldom required where adhesive methods are being planned

- Conventional restorations are associated with higher initial financial costs - perhaps three fold that of adhesive restorations (in the experience of the authors)

- Conventional restorations may offer superior levels of longevity when compared to adhesive restorations (as discussed below); however failures in the longer term tend to be catastrophic and often un-amenable to repair. ${ }^{8}$ In contrast, failures associated with adhesive restorations used to treat wear are often readily addressed, without further biological detriment to the dental hard tissue(s), as will be discussed further in the fourth paper in this series.

In general, it would be prudent (where possible) to primarily consider an adhesive,
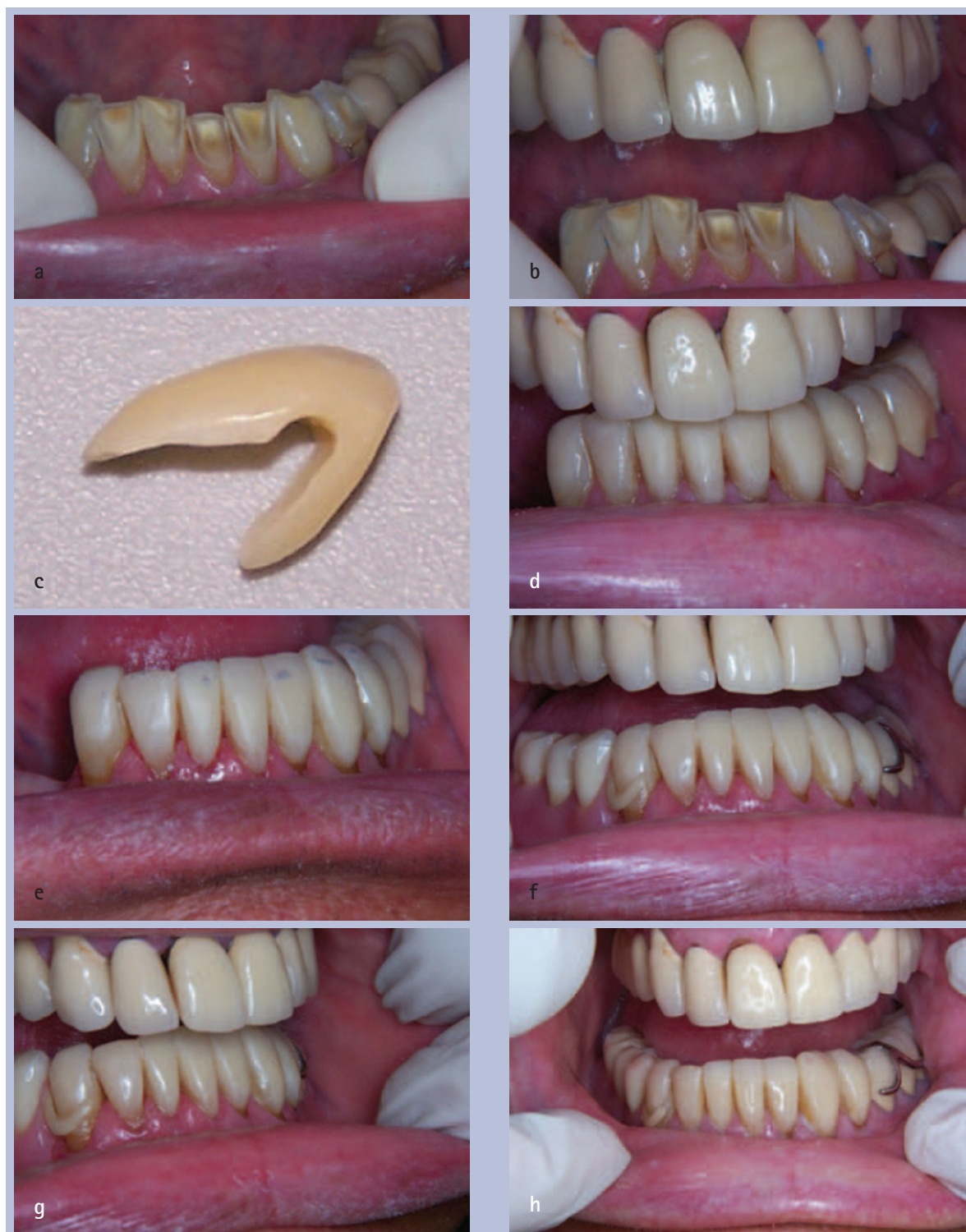

Fig. 2 Clinical case 1. a-b) Pre-operative views of case. c) Close up view of overlay restoration (Ceramage, Shofu). d-e) Cemented overlays and provisional crowns at LL5, 6 and 7. Note the presence of even occlusal contacts in centric occlusion. Provisional restorations were retained for eight weeks to enable assessment of tolerance/adaptability to the new occlusal prescription $\mathrm{f}-\mathrm{h}$ ) Provisional restorations have been replaced with definitive restorations. Edentulous space has been restored with a tooth and mucosally supported lower partial denture. Note the presence of canine guidance on lateral excursive and protrusive mandibular movements

additive approach, when attempting to actively manage a worn dentition. As discussed in the preceding article in this series, ${ }^{2}$ adhesive restorations may very effectively serve as medium term restorations, where eventually they may be replaced with conventional techniques, having established the patient's tolerance and adaptability to their new occlusal scheme (should a non-conformist approach be adopted). In addition, the undertaking of tooth preparations of teeth which have been additively restored by the addition of adhesive materials, may at times largely involve removal of the restorative material (as opposed to dental hard tissues).

\section{CATEGORY 1 PATIENTS: 'EXCESSIVE TOOTH WEAR, TOGETHER WITH A LOSS IN THE OVD'}

Such cases may be considered the most straightforward of all three categories to manage, as the resultant inter-occlusal clearance created through the process of tooth wear will provide most, if not all the required space for the restorative material, without the need for aggressive occlusal reduction (by a planned increase in the OVD), while maintaining a physiological Freeway Space (FWS). ${ }^{9}$

A full coverage, hard acrylic stabilisation splint, such as a Michigan splint, can be used to evaluate the patient's tolerance/ 
adaptability to the planned occlusal changes (as described in further detail in paper $1^{1}$ ). This should be fabricated to not only provide the planned increase in OVD, but also the occlusal prescription as described above, hence a mutually protective occlusal scheme. For category 1 patients however, the use of such a splint is usually not considered to be mandatory. ${ }^{9}$ Where an adhesive re-constructive approach is planned, all the involved teeth can be potentially prepared in one visit. It would be hoped that tooth preparation will be confined to the application of finishing lines to the affected teeth to assist with the fabrication and placement of any indirect restorations, and also possibly help to augment resistance form. Pre-existing amalgam and composite resin restorations should be replaced with new resin based materials, to improve bonding. Preparation designs for the latter forms of restoration have been detailed further in the preceding article of this series, however, the importance of a copious quantity of high quality tooth enamel and the attainment of meticulous moisture control cannot be overemphasised (in order to attain a successful restorative outcome).

A new facebow record and intra-occlusal record may be required. Where it may be decided to sequence the placement of restorations, such that either the anterior or posterior segments are restored in separate visits, the use of a stabilisation splint (which may be sectioned to accommodate the new restorations) can prove vital to ensure short term longevity of the new restorations, by providing some level of protection from excessive occlusal loads.

Ideally, half the increase in OVD should be incorporated into each arch, but this depends on the pattern of wear, and the desired aesthetic outcome. Where the increase in OVD is shared equally between the dental arches, it will not only allow for a better distribution of the increase in crown to root ratio, but also make the increase in OVD less abrupt, thereby improving the chances of successful adaptation. $^{7}$

Clinical case 1, Figure 2, is an example of a category 1 patient treated via an adhesive, additive approach. It is a case of a 67-year-old male, presenting with an edentulous space in his lower right quadrant, and wearing lower anterior dentition,
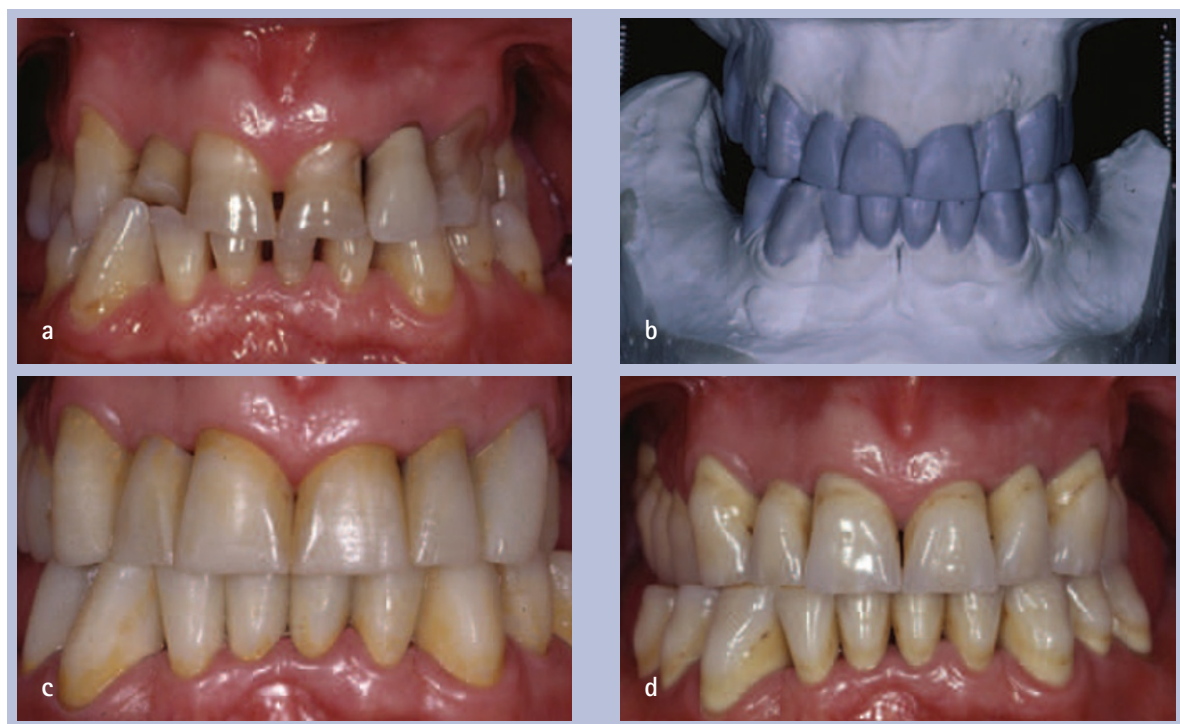

Fig. 3 Clinical case 2. Generalised tooth wear case treated by the means of conventional porcelain fused to metal crowns. a) Pre-operative view of category 1 generalised wear case with loss of OVD. b) Diagnostic wax up. c) Laboratory fabricated provisional restorations. d) Porcelain fused to metal restorations fabricated to copy the occlusal and aesthetic prescription derived from the placement of provisional restorations

This case was completed several years ago. The authors would not advocate this method of treatment provision in current times (in the first instance) given the pre-existing dental condition, as it would be considered to be a highly invasive, aggressive approach

with an FWS of $6 \mathrm{~mm}$. The likely cause of tooth wear in this case is a combination of a bruxist tendency, which has been accentuated in the lower anterior region by the presence of an abrasive antagonistic porcelain fused to metal crowns (with ceramic on the occluding surface[s]) The upper arch had been previously restored entirely by the means of porcelain-fused to metal crowns and bridges. Metalloceramic crowns were present at LL5, 6 and 7. It was decided to restore the worn lower anterior teeth (LL321, LR123) with indirect resin ceromer overlays (Ceramage, Shofu), and to concomitantly increase the OVD by $3 \mathrm{~mm}$. The first stage involved the elective removal of the crowns at LL5, 6 and 7. This was followed by the placement of the composite onlays (luted with Variolink II, Ivoclar, Vivadent) and the fitting of provisional crowns at LL5, 6 and 7 to the new occlusal vertical height. After a period of adaptation, the provisional crowns were replaced with definitive metallo-ceramic crowns. The crowns were designed so as to include rest seats, guide planes and undercuts in accordance to the partial denture design. The restorations were designed to provide a canine guided occlusion, with even centric stops in $\mathrm{CO}$ and posterior disclusion on protrusion and lateral excursions.
Where conventional restorations are being planned, preliminary tooth preparations (of at least one arch) can be carried out in one single visit. This will allow for the fabrication of the provisional restorations for all the teeth at the planned OVD. The choice of which arch to prepare first will depend on the occlusal plane discrepancy (usually the arch with the greatest discrepancy will be prepared first). Acrylic or silicone indices formed from the diagnostic wax up can be used to assist the operator with the level of occlusal reduction required.

The patient should be maintained in indirectly formed provisional restorations for a period of 6-8 weeks. ${ }^{9}$ This time period will allow for an evaluation of the aesthetics and function. The provisional restorations may, however, require adjustments. Once deemed acceptable, the provisional restorations may be used as an occlusal and aesthetic guide for the fabrication of the definitive restorations. The construction of a customised anterior guidance table can prove to be very beneficial (to copy the anterior occlusal scheme) as achieved with the use of the provisional restorations), where the anterior guidance has been shown to be mechanically acceptable.

Where metallo-ceramic crowns are being prescribed, it may be worthwhile 
undertaking either metal and/or biscuit try-in stages, so as to minimise the risk of errors propagating during the undertaking of restorative care. The final occlusal scheme should aim to leave the patient with a mutually protected occlusion as discussed above.

Clinical case 2, Figure 3, is an example of a category 1 patient treated by the means of conventional restorations. Please note, this approach given the state of the pre-existing dental health is not advocated by the authors (as a first line means for tooth wear management), as it is considered to be biologically highly invasive.

\section{CATEGORY 2: 'EXCESSIVE WEAR WITHOUT LOSS OF OVD, BUT WITH LIMITED SPACE AVAILABLE'}

In such cases, a discrepancy will usually exist between centric occlusion (CO) and centric relation (CR). CR may provide space to accommodate restorative materials; however, it might not always be fully adequate and there may be a need to plan an increase in the OVD. For such cases, the patient should be provided with a full coverage, hard acrylic occlusal splint, which will provide an increase in the OVD to the required range, while the mandible is manipulated into its retrusive arc of closure. ${ }^{9}$

The occlusal prescription of the splint should aim to provide a removable mutually protective scheme. The patient should be instructed to wear the splint continually for a period of one month (at all times other than when eating) to evaluate the tolerance of the increase in OVD.

Once the operator is satisfied that the patient can tolerate the planned change, the process of preliminary tooth preparation may begin, as described for category 1 patients.

Unpredictable compliance with splint therapy has prompted an alternative approach, as described by Vialati and Belser in 2008. ${ }^{10}$ The latter have suggested that a more realistic approach would involve the placement of indirect provisional resin composite onlay and/ or palatal resin veneers respectively at the same occlusal prescription as would be provided by a full coverage, hard occlusal splint. The 'true reversibility' of this approach may, however, be a concern (as some level of tooth reduction may
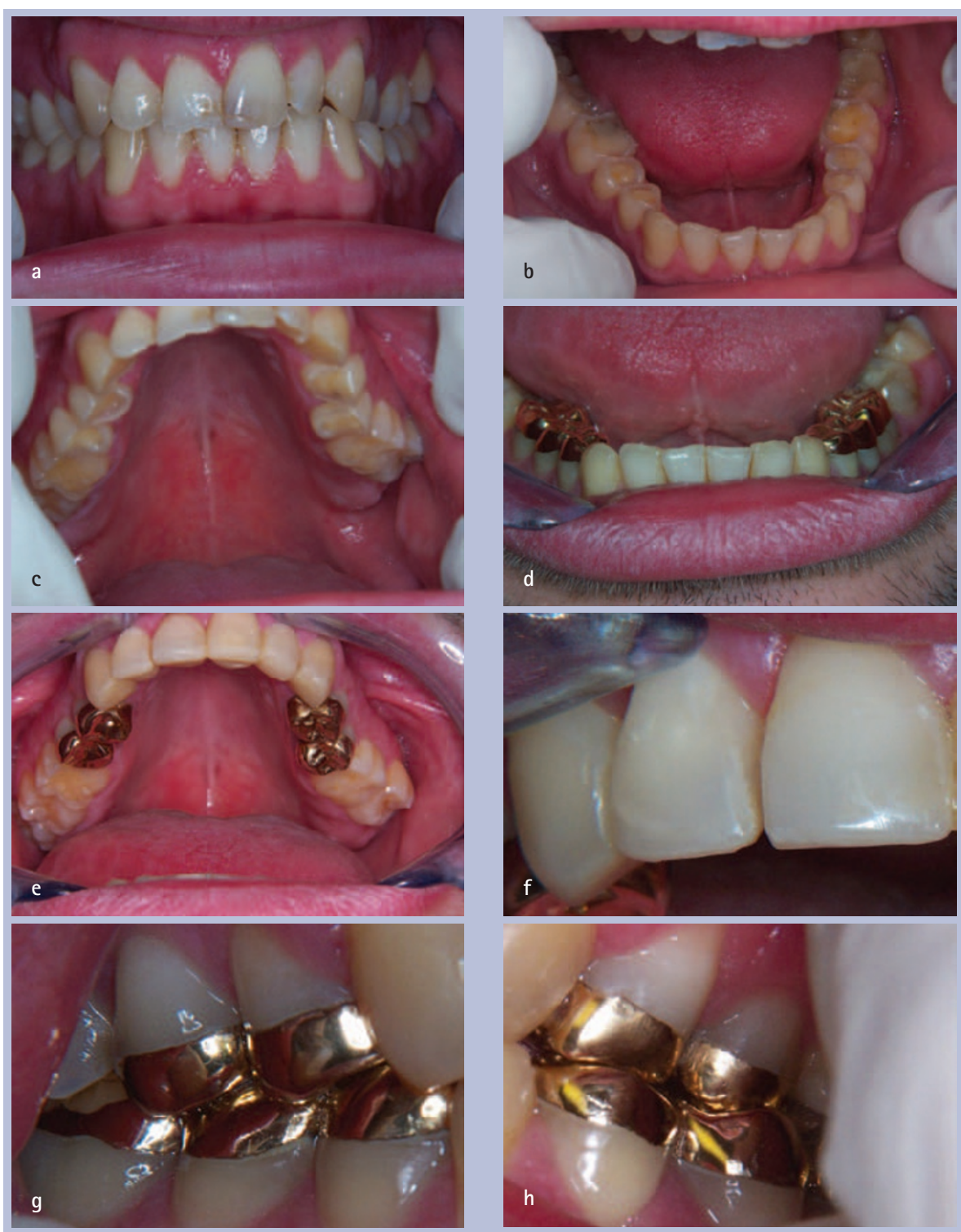

Fig. 4 Clinical case 3. a-c) Pre-operative views of case. $d$-h) Post-operative views. Worn occlusal surfaces have been restored with cast gold adhesive onlays posteriorly, while the maxillary anterior teeth have been restored with indirect resin composite restorations. The placement of these restorations culminated in a very small separation of the second molar teeth. These teeth were allowed to 'Dahl' into occlusion

be required to accommodate the desired thickness of composite resin) as may be the additional financial costs incurred. While resin based restorations may be applied 'directly' to reduce costs, this approach is very time and skill demanding and indeed, it may be impossible to attain the desired dynamic occlusal scheme particularly for complex posterior restorations, which will need to be placed in a supra-occlual position (at the desired new vertical dimension).

Clinical case 3, Figure 4, involves that of a 31-year-old male patient who presented with generalised TW due to a combination of bruxism and extrinsic erosion. While a discrepancy was elicited between $\mathrm{CO}$ and $\mathrm{CR}$, there was inadequate inter-occlusal clearance to accommodate restorations without maintaining a physiological FWS. A Michigan splint was provided to increase the OVD by $3.5 \mathrm{~mm}$. Following a period of successful adaptation (for one month), the patient's dentition was restored by adhesive means, with minimal tooth preparation. The posterior teeth were managed by the application of Type III cast gold adhesive onlays, where the fit surface had been heat treated at $400^{\circ} \mathrm{C}$. The onlays were cemented using Panavia 2.0F (Kuraray, Japan). The maxillary anterior dentition was restored by the means of indirect resin ceromer (Ceramage, Shofu) palatal veneers with 
incisal edge coverage (cemented using Variolink II). It is worth noting is that there was little need to restore the second molar teeth or lower incisor teeth. The former were allowed to re-establish occlusal contacts through the combined processes of mandibular repositioning and the controlled intrusive and extrusive movements of involved dento-alveolar processes respectively.

\section{CATEGORY 3: 'NO LOSS OF OVD, WITH INSUFFICIENT SPACE FOR RESTORATIVE MATERIALS}

A typical example of a category 3 case is shown by Figure 5.

These are usually the most difficult cases to restore because space is not readily available due to tooth repositioning brought about by alveolar compensatory growth. According to Rivera-Morales and Mohl, ${ }^{9}$ for such cases, every effort should be made to obtain space by means other than an increase in the OVD. Only if such methods fail to provide enough space, would an increase in the OVD be advocated. The latter would be accomplished by the programmed modification of the OVD through the careful use of occlusal splints.

Other methods which may be used to create space include:

\section{Surgical crown lengthening, with} osseous re-contouring. Surgical crown lengthening with osseous re-contouring can be used to increase the quantity of coronal tooth tissue, particularly in the case of teeth with short clinical crown heights, where further occlusal reduction may severely compromise retention and resistance form where conventional restorations are being planned.

While restoration margins may be placed subgingivally, ideally the restoration margin should be placed no more than $0.5 \mathrm{~mm}$ subgingivally to prevent encroachment of the biological width. Where the latter is invaded, chronic periodontal inflammation with concomitant periodontal breakdown will follow, permitting the re-establishment of the biological width at a more apical position. To avoid this, surgical crown lengthening is indicated (Briggs and Bishop, 1993 ${ }^{11}$ ). However, surgical crown lengthening may
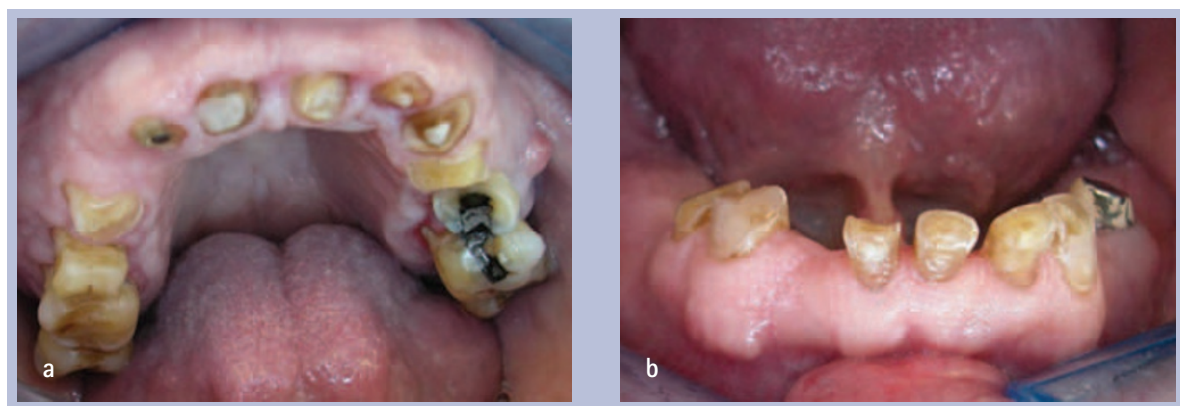

Figs 5a-b Example of a category 3 case

result in unsightly 'black triangles' between the teeth and also lead to unfavourable crown to root ratios. ${ }^{11}$ Gingival recession often accompanies the healing process, which may result in the exposure of subgingival margins. Where possible, methods of tissue retraction and impression making should avoid damage to the supra-crestal attachment (Talbot et $a l .{ }^{12}$ ). It is also important to allow an adequate period of healing before the placement of definitive restorations (particularly in the anterior region), for the avoidance of poor post-restorative aesthetics and to allow the level of the gingival crest to stabilise. ${ }^{12} \mathrm{~A}$ period of up to six months between that of the undertaking of periodontal surgery to that of the placement of the definitive restorations has been advocated by Wise. ${ }^{13}$

Other drawbacks associated with crown lengthening include significant post-operative sensitivity, especially as the restorative margins will need to be placed up on newly exposed root dentine. ${ }^{11}$ Where the treated tooth has a marked coronal-cervical taper, this may also culminate in the need for a highly destructive preparation form. The presence of high furcal areas must also be considered pre-operatively.

Figure 6 shows an example of a lower anterior dentition displaying signs of reduced clinical crown height due to pathological tooth wear. Clinical crown lengthening has been achieved by the means of a conventional periodontal surgical approach.

2. Elective endodontics. Elective endodontics may be considered to permit the application of a post and core system to further augment the
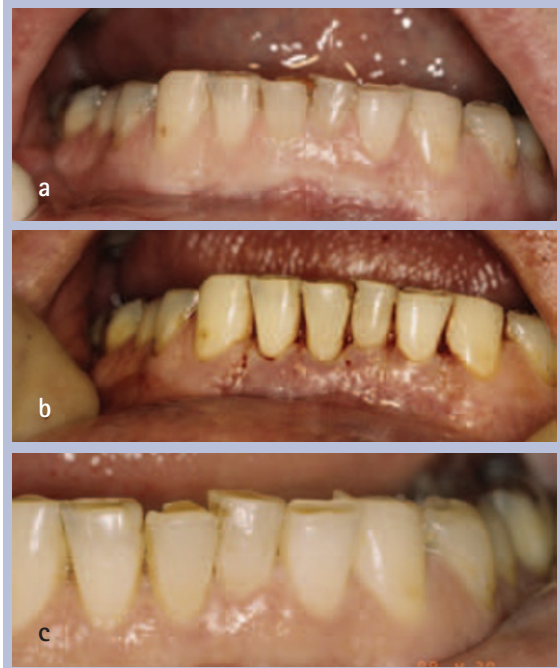

Fig. 6 a-b) Limited crown height of lower anterior teeth. c) Increased crown height after healing has taken place

available core material, or in the case of a grossly over-erupted tooth, where there is a need to correct the occlusal plane discrepancy (where occlusal reduction would otherwise result in iatrogenic pulpal exposure). However, as discussed in paper 2 of this series, ${ }^{2}$ post and core restorations where there may be signs of wear as a result of parafunctional tooth clenching or grinding habits may be associated with a very bleak outlook. ${ }^{14}$ Furthermore, the undertaking of elective endodontic therapy might also compromise the long term prognosis of the affected tooth, should root canal therapy prove to be unsuccessful

3. Orthodontic tooth movement. Orthodontic tooth movement can also be used to permit the intrusion of grossly over-erupted teeth or the extrusion of teeth with short clinical crowns (where there is a copious quantity of alveolar bone support). ${ }^{15}$ particularly when placed in dentitions 


\section{SUMMARY}

The provision of active restorative care for a patient presenting with generalised tooth wear can be considerably demanding even for more experienced dental operators. There is an obviously profound prerequisite for the latter to have a very clear perspective of the planned outcome, which in turns requires a very good working knowledge of the principles of occlusion and an appreciation of the shortcomings of available materials and techniques available to the contemporary practitioner. The final article of this four part series of papers on the subject of tooth wear will provide an account of the commonly used materials and techniques which may be applied to treat cases of tooth wear.
1. Mehta S B, Banerji S, Millar B J, Suarez-Feito J-M. Current concepts on the management of tooth wear: part 1. Assessment, treatment planning and strategies for the prevention and the passive management of tooth wear. Br Dent J 2012; 212: 17-27.

2. Mehta S B, Banerji S, Millar B J, Suarez-Feito J-M Current concepts on the management of tooth wear: part 2. Active restorative care 1: the management of localised tooth wear. Br Dent J 2012; 212: 73-82.

3. Turner $K$, Missirilian D. Restoration of the extremely worn dentition. J Prosthet Dent 1984; 52: 467-474.

4. Rosenstiel S, Land M, Fujimoto J. Contemporary fixed prosthodontics. 3rd ed. Mosby, 2011.

5. Staurt C, Stallard H. Concepts of occlusion. Dent Clin North Am 1963; 7: 591.

6. Saunders W, Saunders E. Prevalence of peri-radicular periodontitis associated with crowned teeth in an adult Scottish subpopulation. Br Dent J 1998; 185: 137-140.

7. Edelhoff D, Sorenssen J. Tooth structure removal associated with various preparation designs for anterior teeth. J Prosthet Dent 2002; 87: 503-509.

8. Smales R, Berekally T. Long term survival of direct and indirect restorations placed for the treatment of advanced tooth wear. Eur J Prosthodont Restor Dent 2007: 15: 2-6.

9. Rivera-Morales W, Mohl N. Restoration of the vertical dimension of the occlusion in the severely worn dentition. Dent Clin North Am 1993; 36: 651-663.

10. Vialati F, Belser C. Full mouth adhesive rehabilitation of a severely eroded dentition: The three step technique. Part 2. Eur J Esthet Dent 2008; 3: 128-146.

11. Briggs P, Bishop K. Fixed prosthesis in the treatment of tooth wear. Eur J Prosthodont Restor Dent 1997; 4: $175-180$.

12. Talbot T R, Briggs P F, Gibson M T. Crown lengthening: a clinical review. Dent Update 1993; 20: 301, 303-306

13. Wise M. Periodontal surgery. In Failure in the restored dentition: management and treatment. Chapter 20. pp 317-334. Quintessence Books, 1995.

14. Mehta S B, Millar B J. A comparison of the survival of fibre posts cemented with two different resin systems. Br Dent J 2008; 205: E23.

15. Wise M. Orthodontic techniques. In Failure in the restored dentition: management and treatment. Chapter 24. pp 353-366. Quintessence Books, 1995. 